\title{
A controlled study of consumption of $\beta$-glucan-enriched soups for 2 months by type 2 diabetic free-living subjects
}

\author{
Christine Cugnet-Anceau ${ }^{1,2,3,4,5,6,8} \dagger$, Julie-Anne Nazare ${ }^{1,2,3,4,5,6} \dagger$, Maria Biorklund ${ }^{7}$, Elodie Le Coquil ${ }^{8}$, \\ Agnès Sassolas ${ }^{9}$, Monique Sothier ${ }^{1,2,3,4,5,6}$, Jorgen Holm ${ }^{10}$, Mona Landin-Olsson ${ }^{11}$, Gunilla Önning ${ }^{7}$, \\ Martine Laville $1,2,3,4,5,6$ and Philippe Moulin ${ }^{1,2,3,4,5,6,8 *}$ \\ ${ }^{1}$ Centre de Recherche en Nutrition Humaine de Rhône-Alpes, F-69495 Pierre-Bénite, France \\ ${ }^{2}$ Université de Lyon (Univ Lyon), F-69622 Lyon, France \\ ${ }^{3}$ INSA de Lyon, RMND, F-69621 Villeurbanne, France \\ ${ }^{4}$ IMBL ou INSERM U870, Faculté Lyon-Sud, F-69921 Oullins, France \\ ${ }^{5}$ INRA U1235, Faculté Lyon-Sud, F-69921 Oullins, France \\ ${ }^{6}$ Hospices Civils de Lyon, Endocrinologie, diabètes et nutrition/hôpital Edouard Herriot, F-69437 Lyon, France \\ ${ }^{7}$ Biomedical Nutrition, Center for Chemistry and Chemical Engineering, Lund University, SE-22100 Lund, Sweden \\ ${ }^{8}$ Fédération d'endocrinologie, maladies métaboliques, diabète et nutrition, Hôpital cardiovasculaire et pneumologique Louis \\ Pradel, F-69677 Bron Cedex, France \\ ${ }^{9}$ Laboratoire de Biochimie, Centre de Biologie Est, 69677 Bron, France \\ ${ }^{10}$ Findus $A B, 26781$ Bjuv, Sweden \\ ${ }^{11}$ Department of Endocrinology and Diabetology, University Hospital, S-221 85 Lund, Sweden
}

(Received 9 March 2009 - Revised 3 July 2009 - Accepted 7 August 2009 - First published online 28 September 2009)

\begin{abstract}
Type 2 diabetes is associated with a higher cardiovascular risk and there has been a growing interest in using dietary intervention to improve lipid profile and glucose control. The present work aims at analysing the effects of the enrichment of a normal diet with $\beta$-glucan ( $3.5 \mathrm{~g} / \mathrm{d})$ in free-living type 2 diabetic subjects for 2 months, using a palatable soup. This trial was a parallel, placebo-controlled, double-blinded randomised study performed in fifty-three type 2 diabetic subjects. During a 3-week run-in period, subjects daily consumed a ready meal control soup (without $\beta$-glucan). For the following 8 weeks, subjects were randomly assigned to consume daily either a control soup or a $\beta$-glucan soup. Changes in lipid profile (total cholesterol (TC), HDL- and LDL-cholesterol (HDLc and LDLc), apo B and TAG) and in glucose control (HbA1c and fasting glucose) were measured. There was no significant alteration in lipid profile in the two groups (TC, HDLc, LDLc and apo B). TAG decreased significantly in the $\beta$-glucan group compared with the control group $(-0.12$ (SD 0.38) v. 0.12 (SD 0.44$)$ mmol/l, $P=0.03$ ). HbA1c and fasting glucose were not reduced in any group. A single daily ingestion of $3.5 \mathrm{~g} \beta$-glucan, as required by official dietary recommendations, for 8 weeks did not change the lipid profile and HbA1c in type 2 diabetic subjects. To improve the metabolic profile of type 2 diabetic subjects in the long term, the quantity, the food vectors and the tolerability of $\beta$-glucan products may be re-evaluated.
\end{abstract}

乃-Glucan: Lipid profile: HbA1c: Type 2 diabetes: Long-term intervention

Type 2 diabetes is associated with a high risk of CVD; consequently, the improvement of lipid profile or blood glucose control is a major challenge. Epidemiological and short-term intervention studies have highlighted that high dietary fibre intake is related to an improvement in fasting and postprandial glycaemic control and lipid profile, associated with a lower cardiovascular risk ${ }^{(1-3)}$. In this context, soluble dietary fibre supplementation has been considered as a therapeutic tool to improve these parameters.

$\beta$-Glucan, a non-starch viscous polysaccharide derived from oat, has been shown to reduce total cholesterol (TC) and LDLc levels, and post-prandial glucose and insulin response $\mathrm{e}^{(4-7)}$. Several mechanisms are proposed as to explain the cholesterol-lowering effect of $\beta$-glucan: reduced cholesterol absorption due to an increased meal bolus viscosity, reduced cholesterol synthesis due to an increased conversion of cholesterol to faecal bile acids and/or an inhibition of hepatic fatty acid synthesis by products of fermentation $^{(8-10)}$. Concerning glucose metabolism, the beneficial metabolic effects of oat $\beta$-glucan are tightly linked to the $\beta$-glucan-induced increased viscosity of the meal bolus, which delays and/or reduces carbohydrates absorption ${ }^{(11,12)}$.

Abbreviations: LDLc, LDL cholesterol; TC, total cholesterol.

$\dagger$ C. C.-A. and J.-A. N. are both first authors of the present paper.

* Corresponding author: Professor Philippe Moulin, fax +33 4726813 07, email philippe.moulin@chu-lyon.fr 
However, the increase in intestinal viscosity is dependant of $\beta$-glucan molecular weight and solubility, food processing, $\beta$-glucan concentration and associated food matrix. $\beta$-Glucan enrichment of food products has been largely studied, because it permits a higher intake per serving with a minimum decrease in palatability ${ }^{(13)}$. So, to reach the $3 \mathrm{~g} / \mathrm{d}$ intake recommended by Food and Drug Administration, consumers must be offered a range of palatable and sufficiently enriched products. Up to now, few studies have specifically focused on the effect of $\beta$-glucan as an adjunct therapeutic aid for metabolic control in type 2 diabetic subjects in the mid-term range ${ }^{(14)}$. In parallel to the numerous epidemiological and short-term intervention studies concerning dietary fibre and diabetes, evidence-based studies investigating long-term efficiency of $\beta$-glucan enrichment in type 2 diabetic subjects are missing.

The present work aims at analysing the effects of the enrichment of a normal diet with reasonable amount of $\beta$-glucan $(3.5 \mathrm{~g} / \mathrm{d})$ on glucose control (HbA1c level) and on lipid profile (TC, HDL cholesterol, LDLc and apo B) for 2 months in a representative group of free-living type 2 diabetic subjects, with different treatments (anti-diabetic, lipid-lowering, diet) and characteristics (time from diagnosis, sex and age). Using a palatable ready-to-eat frozen soup seemed to be a good alternative to maintain both physiological effects and good sensory properties and to implement an everyday dietary intervention over a long-term period.

\section{Subjects and methods}

\section{Study design}

The present study was a parallel, placebo-controlled, blinded randomised trial. For a run-in period of 3 weeks, the subjects consumed one control soup a day (without $\beta$-glucan). For the following 8 -week intervention period, one group continued to consume the control soup (control group), while the other group consumed the soup containing $3.5 \mathrm{~g} \beta$-glucan ( $\beta$-glucan group).

At the beginning of the run-in period (week (W) 0), of the dietary intervention (W3) and at the end (W11), body weight was measured and fasting blood samples were collected. The subjects completed a 5-d dietary questionnaire on W0 and W11.

\section{Study population}

The type 2 diabetes patients were recruited in Lund and Lyon, and a medical examination and screening visit were performed prior involvement, received written and oral information about the protocol and signed an informed consent form. The study was approved by the Scientific Ethics Committees in Lyon (CCPPRB Lyon A) and Lund and accorded with both the French 'Huriet-Serusclat' law and the Second Declaration of Helsinki. The inclusion criteria were men or women aged $30-75$ years old, BMI $20-35 \mathrm{~kg} / \mathrm{m}^{2}$, stable body weight, with an $\mathrm{HbA} 1 \mathrm{c}<11 \%$. The exclusion criteria were pregnancy, breastfeeding, severe renal complications, secondary dyslipidaemia, TAG $>4 \mathrm{mmol} / \mathrm{l}$, anaemia, treatment by orlistat, pancreatic disease and malignancy $>1$ year ago.

\section{Experimental products and dietary regimen}

The experimental products were frozen ready-to-eat soups (Findus R\&D, Bjuv, Sweden) with three different flavours: lentil and ham; shrimps and dill; mushroom (composition in Table 1). A soluble oat concentrate of $\beta$-glucan ( $3.5 \mathrm{~g}$ ) was added to the enriched soups (Ceba Foods, Lund, Sweden). Attempts were made to increase further the concentration of $\beta$-glucan, but this negatively affected the palatability. The soups composition has been adjusted to obtain levels with respect to carbohydrates and lipids, by adding rapeseed oil to control soup and maltodextrin to $\beta$-glucan soup. $\beta$-Glucan in the soup has a molecular weight of $80 \mathrm{kDa}$, and preparation, freezing and storage did not alter the molecular weight (analysis by VTT Biotechnology, Espoo, Finland).

The subjects were instructed to consume daily one soup as the main component of a lunch or dinner meal and if desired add drink and/or other food products. Subjects and investigators were blinded for the type of soup ingested.

As a measure of compliance, packages that were left over must be returned and were counted.

Subjects were also asked not to change their usual food habit, physical activity and to record any signs of illness, medication used or deviation to the protocol.

Food habits were assessed once before the run-in period and once at the end of the intervention by a 5-d food intake questionnaire and analysed by a trained registered dietitian (in Lyon, using a SUpplémentation en VItamines et Minéraux AntioXydants $(\mathbb{C}$ dietary photographic support and in Lund, a food database from the Swedish National Food Administration (PC-Kost 1_99, SLV, Uppsala, Sweden).

\section{Testing and analytical procedures}

Subjects came to the research centre at the start of the study (W0), at the end of the run-in period (W3), at the middle of

Table 1. Nutrient composition of the soups (per $100 \mathrm{~g})^{\star}$

\begin{tabular}{|c|c|c|c|c|c|c|}
\hline \multirow[b]{2}{*}{ Nutrients } & \multicolumn{2}{|c|}{ Lentil soup } & \multicolumn{2}{|c|}{ Shrimps and dill soup } & \multicolumn{2}{|c|}{ Mushroom soup } \\
\hline & $\beta$-Glucan soup & Control soup & $\beta$-Glucan soup & Control soup & $\beta$-Glucan soup & Control soup \\
\hline Energy (kJ) & 351.9 & 331.0 & $213 \cdot 7$ & 196.9 & $301 \cdot 7$ & $280 \cdot 7$ \\
\hline Protein $(\mathrm{g})$ & 3.8 & 2.6 & 3.7 & 2.6 & 3.1 & $2 \cdot 0$ \\
\hline Fat $(\mathrm{g})$ & 4.8 & $4 \cdot 8$ & $2 \cdot 0$ & $2 \cdot 0$ & 3.0 & 3.0 \\
\hline Carbohydrate (g) & $6 \cdot 4$ & 6.4 & 4.6 & 4.6 & $8 \cdot 1$ & 8.1 \\
\hline Total fibre (g) & $2 \cdot 3$ & $1 \cdot 1$ & 1.9 & 0.7 & $2 \cdot 5$ & 1.4 \\
\hline$\beta$-Glucan (g) & 0.88 & 0 & 0.88 & 0 & 0.88 & 0 \\
\hline
\end{tabular}

* One portion serving is $400 \mathrm{~g}$. 
the study (W7) and at the end of the study (W11). Body weight was measured using a calibrated scale. Blood samples were drawn following an overnight fast, collected in tubes maintained at $4^{\circ} \mathrm{C}$ and immediately centrifuged at $4500 \mathrm{rpm}$ for $10 \mathrm{~min}$ at $4^{\circ} \mathrm{C}$. Plasma was then stored at $-20^{\circ} \mathrm{C}$ until assay.

Fasting glucose concentrations, HDL cholesterol, TC and TAG were analysed with respective enzymatic kits from Roche Diagnostics using an autoanalyzer (Roche Diagnostics Hitachi 917; Hitachi, Tokyo). LDLc was calculated by the Friedewald formula ${ }^{(15)}$. Plasma concentrations of apo B were determined by immunonephelometry using an Immage Beckman instrument (Beckman Instruments, Fullerton, CA, USA). HbA1c levels were determined by HPLC (Biorad, Marnes la Coquette, France).

\section{Statistical analysis}

Results are given as means and standard deviations except TC, LDLc, HbA1c and fasting glucose when plotting in graphs (standard error of the mean). Changes in parameters according to group assignment were calculated as the change and the percentage of change between values at the end of the run-in period (W3) and at the end of the intervention period (W11).

Equality of variance and distribution normality have been checked prior further analysis. Differences between groups at baseline and change and percentage of change between $\mathrm{W} 3$ and W11 were assessed using an unpaired $t$ test. In case of non-normal distribution, a non-parametric Mann and Whitney test was done. The present study including thirty subjects in each group has the power to detect a significant decrease in Hbalc lower or equal to $0.5 \%$ with a standard deviation $=0.7 \% \quad$ ( $\beta$-risk of $20 \%$ and $\alpha$-risk of $5 \%$; P Moulin, unpublished clinical). Statistical significance was inferred at $P<0.05$. All statistical analyses were performed using Statview version 5.0 (SAS Institute, Cary, NC, USA) software.

\section{Results}

\section{Subjects}

Sixty-seven diabetic volunteers were screened in Lyon and Lund and were randomly assigned to either the control group or the $\beta$-glucan group. There was no centre effect for any studied parameter. Only fifty-three subjects (twenty-four subjects in the control group and twenty-nine subjects in the $\beta$-glucan group) were included in the analysis due to lipid profile anomaly (two subjects: TAG $>4 \mathrm{mmol} / \mathrm{l}$ at W0) or withdrawal (twelve subjects). The population was composed of twenty-one women and thirty-two men with mild obesity. About $50 \%$ of the subjects were under lipid-lowering treatment (twelve in the control group and fifteen in the $\beta$-glucan group). As for anti-diabetic treatment, subjects were either under insulin or medication treatment (twelve in the control group and nine in the $\beta$-glucan group), either under diet and/or medication treatment (twelve in the control group and twenty in the $\beta$-glucan group). Importantly, the treatments were kept constant throughout the study. The subjects' characteristics are given in Table 2.
Table 2. Baseline characteristics of the study subjects according to group

(Mean values and standard deviations)

\begin{tabular}{|c|c|c|c|c|}
\hline & \multicolumn{2}{|c|}{ Control group ( $n$ 24) } & \multicolumn{2}{|c|}{$\beta$-Glucan group $(n$ 29) } \\
\hline & Mean & SD & Mean & SD \\
\hline Age (years) & $61 \cdot 8$ & 7.5 & 61.9 & 9.1 \\
\hline Body weight $(\mathrm{kg})$ & 86.41 & $29 \cdot 02$ & $87 \cdot 32$ & $17 \cdot 23$ \\
\hline BMI $\left(\mathrm{kg} / \mathrm{m}^{2}\right)$ & 29.02 & 4.05 & 30.48 & 4.08 \\
\hline $\mathrm{HbA1c}(\%)$ & 7.47 & $1 \cdot 29$ & $7 \cdot 31$ & 0.92 \\
\hline $\begin{array}{l}\text { Fasting glucose } \\
\qquad(\mathrm{mmol} / \mathrm{l})\end{array}$ & $8 \cdot 36$ & $2 \cdot 28$ & 8.84 & $2 \cdot 11$ \\
\hline $\mathrm{TC}(\mathrm{mmol} / \mathrm{l})$ & 4.70 & 0.81 & 4.93 & 1.08 \\
\hline $\mathrm{HDLc}(\mathrm{mmol} / \mathrm{l})$ & $1 \cdot 18$ & 0.37 & $1 \cdot 17$ & 0.34 \\
\hline LDLc (mmol/l) & $2 \cdot 87$ & 0.70 & $2 \cdot 87$ & 0.90 \\
\hline TAG (mmol/l) & 1.29 & 0.46 & $1.77^{\star}$ & 0.81 \\
\hline apo B (mmol/l) & 0.95 & 0.16 & 0.99 & 0.25 \\
\hline CRP (mg/l) & 3.32 & 3.25 & $3 \cdot 15$ & 3.45 \\
\hline
\end{tabular}

TC, total cholesterol; HDLc, HDL cholesterol; LDLc, LDL cholesterol; CRP, C-reactive protein.

* Mean values were significantly different between $\beta$-glucan and control groups (Mann-Whitney test; $P=0.02$ ).

They were normocholesterolaemic with a mild hypertriglyceridaemia and a fair blood glucose control. Due to randomisation, both groups were comparable, except for a higher concentration of plasma TAG in the $\beta$-glucan group.

\section{Side effects and compliance}

All the participants followed the experimental protocol without difficulty. The compliance was good (based on soup packages count). The soup portion was mostly ingested at dinner in the two groups.

There were no significant differences between the groups in the safety parameters: number of results leucocytes, erythrocytes and platelets; or serum concentration of CRP, alanine aminotransferase, aspartate aminotransferase, $\gamma$-glutamyltransferase and creatinine (data not shown). There was no record of side effects as headache, stomach complaints, nausea, flatulence, diarrhoea, bloated feeling, eruption/rashes, fatigue and dizziness. The twelve withdrawals, with a similar distribution in both groups, were not due to diet intolerance or side effects, but to personal reasons.

\section{Run-in period}

During the 3-week run-in period, none of the measured metabolic parameters was modified in the two groups (data not shown).

\section{Anthropometric parameters and diet}

The mean baseline BMI in both groups (Table 2) remained constant throughout the study and did not significantly differ between groups: $+0.18(\mathrm{SD} 1.33) \mathrm{kg} / \mathrm{m}^{2}$ in the $\beta$-glucan group $v .+0.36(\mathrm{SD} 1.37) \mathrm{kg} / \mathrm{m}^{2}$ in the control group during intervention period $(P=0.63$; data not shown).

The mean daily dietary intakes before the run-in period (W0) were measured: 6.9 (SD 1.6) MJ of total energy; 34.3 (SD 6.2) \% of total energy as fat; 19.0 (SD 2.4) \% of 
total energy as protein; 44.1 (SD 5.4) \% of total energy as carbohydrates; 19.7 (SD 5.3) g of dietary fibres for the $\beta$-glucan group $v$. 8.2 (SD 3.0) MJ of total energy; 35.2 (SD 5.5) $\%$ of total energy as fat; 17.9 (SD 2.7) \% of total energy as protein; 43.7 (SD 7.4) \% of total energy as carbohydrates; 22.3 (SD 12.0) g of dietary fibres for the control group. The registered energy and nutrient intake remained stable throughout the study, and there were no significant differences in either group at the end of the intervention period (W11). However, a trend toward a mild decrease (NS) in fibre consumption during the intervention period was noticed in the two groups.

\section{Serum lipids}

The blood lipid profile was unchanged during the $\beta$-glucan dietary intervention (Table 3). TC and LDLc decreased in the $\beta$-glucan group, but this decrease was NS compared with the control group (Fig. 1(a) and (b)). The apo B concentration remained also stable in both groups. HDL cholesterol increased significantly between $\mathrm{W} 0$ and W11: +5.37 (SD 10.47) \% $(P=0.01)$ in the $\beta$-glucan group. No significant difference was found in HDL cholesterol/LDLc ratio between W3 and W11 in the two groups (data not shown). The serum TAG decreased by -3.75 (SD 22.2) $\%$ in the $\beta$-glucan group and increased by +11.24 (SD 32.40) $\%$ in the control group. This difference between the two groups was significant, even after adjustment for TAG concentration at W0 or W3 as covariant $(P=0 \cdot 03)$. Additionally, the lipid profile's changes were not altered by the presence or absence of a lipid-lowering drug treatment.

\section{Glycaemic control}

The primary glycaemic end point, HbAlc, was not reduced during intervention period in the $\beta$-glucan group (Table 3; Fig. 1(c)). The W11-W3 HbA1C change was not different between the two groups. The mild increase in fasting glucose over the duration of the study was NS and similar in both groups (Table 3; Fig. 1(d)).

Table 3. Change in total cholesterol (TC), LDL- (LDLC), HDL-cholesterol (HDLC), TAG, apo B, HbA1c and fasting glucose*

(Mean values and standard deviations)

\begin{tabular}{|c|c|c|c|c|c|}
\hline & \multicolumn{2}{|c|}{ Control group } & \multicolumn{2}{|c|}{$\beta$-Glucan group } & \multirow[b]{2}{*}{$P$} \\
\hline & Mean & SD & Mean & SD & \\
\hline \multicolumn{6}{|l|}{$\mathrm{TC}(\mathrm{mmol} / \mathrm{l})$} \\
\hline End of run-in (W3) & 4.80 & 0.84 & 4.88 & 1.09 & \\
\hline End of intervention period (W11) & 4.79 & 0.81 & 4.81 & 1.03 & \\
\hline Change & 0.01 & 0.52 & -0.06 & 0.52 & 0.59 \\
\hline Change (\%) & 1.07 & $11 \cdot 10$ & -0.49 & $12 \cdot 64$ & 0.63 \\
\hline \multicolumn{6}{|l|}{$\operatorname{LDLc}(\mathrm{mmol} / \mathrm{l})$} \\
\hline End of run-in (W3) & 2.93 & 0.74 & $2 \cdot 85$ & 0.90 & \\
\hline End of intervention period (W11) & 2.80 & 0.62 & 2.79 & 0.88 & \\
\hline Change & -0.10 & 0.51 & -0.05 & 0.38 & 0.72 \\
\hline Change (\%) & -1.06 & $18 \cdot 86$ & -0.78 & 17.50 & 0.95 \\
\hline \multicolumn{6}{|l|}{$\mathrm{HDLc}(\mathrm{mmol} / \mathrm{l})$} \\
\hline End of run-in (W3) & $1 \cdot 19$ & 0.31 & 1.14 & 0.3 & \\
\hline End of intervention period (W11) & 1.24 & 0.36 & 1.20 & 0.29 & \\
\hline Change & 0.03 & $0 \cdot 14$ & 0.05 & $0 \cdot 10$ & 0.67 \\
\hline Change (\%) & 3.38 & 11.78 & 5.37 & $10 \cdot 47$ & 0.51 \\
\hline \multicolumn{6}{|l|}{ TAG (mmol/l) } \\
\hline End of run-in (W3) & 1.35 & 0.53 & 1.75 & 0.68 & \\
\hline End of intervention period (W11) & 1.47 & 0.65 & 1.63 & 0.55 & \\
\hline Change & 0.12 & 0.44 & -0.12 & 0.38 & $0.03 \dagger$ \\
\hline Change (\%) & 11.24 & $32 \cdot 40$ & -3.75 & $22 \cdot 2$ & $0.05 \dagger$ \\
\hline \multicolumn{6}{|l|}{ apo B (mmol/l) } \\
\hline End of run-in (W3) & 0.93 & $0 \cdot 18$ & 0.98 & 0.24 & \\
\hline End of intervention period (W11) & 0.95 & $0 \cdot 17$ & 0.95 & 0.24 & \\
\hline Change & 0.01 & $0 \cdot 12$ & -0.02 & $0 \cdot 10$ & 0.15 \\
\hline Change (\%) & $3 \cdot 12$ & 13.56 & -2.42 & 11.00 & $0 \cdot 10$ \\
\hline \multicolumn{6}{|l|}{$\mathrm{HbA} 1 \mathrm{c}(\%)$} \\
\hline End of run-in (W3) & $7 \cdot 16$ & $1 \cdot 20$ & $7 \cdot 18$ & 0.92 & \\
\hline End of intervention period (W11) & 7.33 & 1.55 & $7 \cdot 17$ & 0.86 & \\
\hline Change & 0.17 & 0.84 & 0.00 & 0.58 & 0.38 \\
\hline Change (\%) & $2 \cdot 20$ & $11 \cdot 17$ & 0.26 & 7.43 & 0.45 \\
\hline \multicolumn{6}{|l|}{ Fasting glucose $(\mathrm{mmol} / \mathrm{l})$} \\
\hline End of run-in (W3) & 8.28 & $2 \cdot 32$ & 8.98 & 1.83 & \\
\hline End of intervention period (W11) & 9.08 & 3.50 & 9.09 & 1.86 & \\
\hline Change & 0.80 & $2 \cdot 24$ & 0.11 & 1.43 & 0.45 \\
\hline Change (\%) & 9.58 & $25 \cdot 85$ & $2 \cdot 27$ & $16 \cdot 44$ & 0.39 \\
\hline
\end{tabular}

Change (\%), percentage change.

* When the run-in period values were used as covariates, the significance level $(P)$ and interpretation of the results did not change.

$\dagger$ Mean values were significantly different between $\beta$-glucan and control groups $(P<0.05$; Mann-Whitney test or unpaired $t$ test). 
(a)

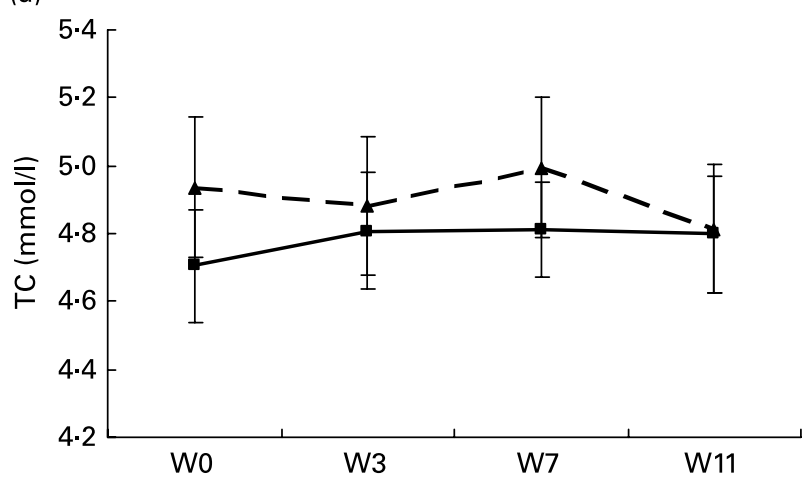

(c)

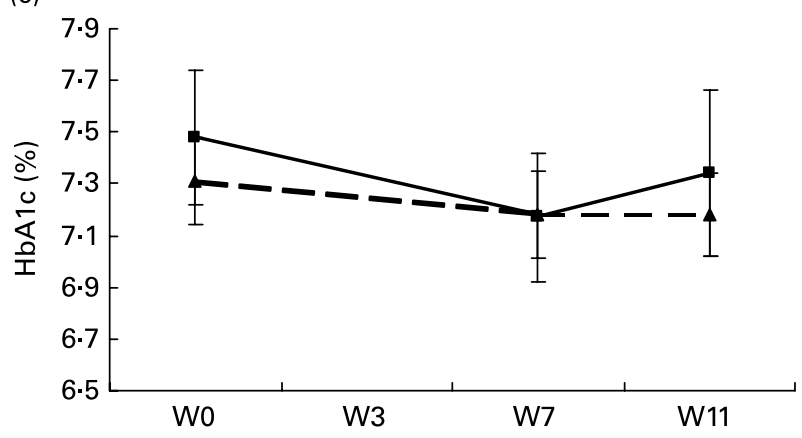

(b)

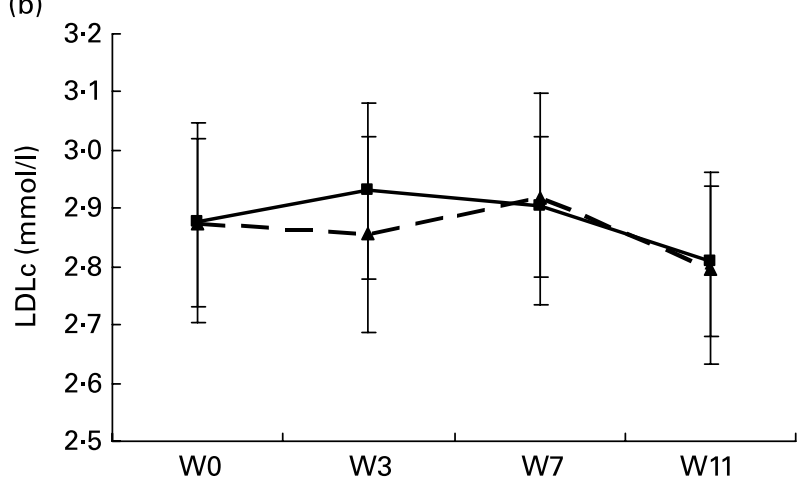

(d)

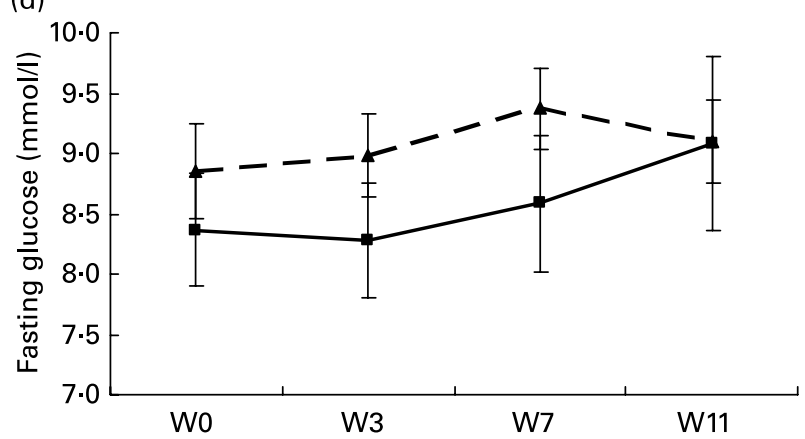

Fig. 1. Mean concentrations with their standard errors of mean during the run-in period (week (W0-W3) and the intervention period (W3-W11) for $\beta$-glucan group ( $\mathbf{\Lambda}$, dotted line; $n$ 29) and for control group (匹, plain line; $n$ 24). (a) Total cholesterol (TC; mmol/l); (b) LDL cholesterol (LDLc; mmol/l); (c) HbA1c (\%); (d) fasting glycaemia (mmol/l). No significant difference was observed between the two groups.

\section{Discussion}

The present study shows that the long-term consumption of a $\beta$-glucan-enriched soup once daily as the main part of the meal had no detectable effect on the metabolic profile in free-living type 2 diabetic subjects. The incorporation of $3.5 \mathrm{~g}$ of $\beta$-glucan in soups, ingested for 8 weeks, did not alter plasma LDLc and HbA1c. The targeted population consisted of a representative group of free-living type 2 diabetes patients. Indeed, the fifty-three subjects (44-75 years old) had a time from diabetes diagnosis ranging between 1 and 25 years. They were under different anti-diabetic treatment (diet, sulphonylurea, metformin, repaglinide and $\alpha$-glycosidase inhibitor) and/or lipid-lowering treatment (fibrates and statins).

The present study was unable to provide evidence of a significant improvement in lipid profile. It has been shown that $3 \mathrm{~g}$ soluble fibre could decrease TC and LDLc ${ }^{(6)}$. According to the meta-analysis of Brown et al. ${ }^{(6)}$, a reduction of $-0.04 \mathrm{mmol} / \mathrm{l}$ per $\mathrm{g}$ of fibre for LDLc would have been expected, so for $3.5 \mathrm{~g}$ of oat $\beta$-glucan $-0.12 \mathrm{mmol} / \mathrm{l}$. In contrast, $\beta$-glucan-enriched beverages (fruit drink and oat milk) did improve the lipid profile in the expected magnitude predicted from this meta-analysis ${ }^{(10,16)}$. However, several other studies failed to demonstrate an effect of $\beta$-glucan incorporation into a solid matrix on the lipid profile in hypercholesterolaemic subjects ${ }^{(17-19)}$. Thus, $5.9 \mathrm{~g} \beta$-glucan incorporated into cookies and bread during 4 weeks did not significantly change TC and LDLc ${ }^{(18)}$. The meta-analysis of Ripsin et al. ${ }^{(20)}$ suggested that a larger reduction of TC and
LDLc were seen in subjects with higher cholesterol levels, particularly when a dose of $3 \mathrm{~g}$ or more of oat fibre was used. However, Biorklund et al. ${ }^{(19)}$ recently has not shown any effect with $4 \mathrm{~g} \beta$-glucan incorporated into a soup in forty-three hyperlipidaemic subjects. Considering the low LDLc encountered in type 2 diabetes subjects, well controlled for lipid profile, the decrease in LDLc was expected to be weaker. Moreover, half of our subjects were treated with a lipid-lowering treatment, which could have blunted the fibre effect, but results were not modified in the subgroup without lipid-lowering treatment (data not shown). Surprisingly, the present results showed for the first time a significant diminution of TAG concentration in the $\beta$-glucan group. It is likely that it is the consequence of the regression toward the mean phenomenon, since the $\beta$-glucan group had a higher level of plasma TAG at baseline.

Few studies have assessed the efficacy of fibres on the lipid profile in type 2 diabetes. In a small group of type 2 diabetic subjects, fed a high amount of soluble fibre ( $7 \mathrm{~g}$ guar gum three times a day for 3 months), a significant reduction in LDLc (from $5.19 \mathrm{mmol} / \mathrm{l}$ (SEM 0.34) to 4.30 (SEM $0 \cdot 40) \mathrm{mmol} / \mathrm{l}$ ) was found ${ }^{(21)}$. Also, another crossover study in eight type 2 diabetic men has shown a decrease in LDLc from 3.36 (SEM 0.12) to 2.59 (SEM 0.12) mmol/l with $9 \mathrm{~g}$ oat bran fibre incorporated into bread, buns or muffins during 12 weeks $^{(22)}$. The higher beneficial effect was observed with ingestion of $8-13 \mathrm{~g}$ konjac-mannan-enriched biscuits associated to a low-fat diet during 3 weeks (LDLc from $3.89(\operatorname{SEM} 0 \cdot 25)$ to $3 \cdot 04(\mathrm{SEM} 0 \cdot 24) \mathrm{mmol} / \mathrm{l})^{(23)}$. 
Concerning glucose control, the anti-diabetic effects of $\beta$-glucans have been suspected in regards to their actions on energy and glucose metabolism and to their lowering effect on post-prandial glycaemic and insulinaemic responses in healthy and fewer in type 2 diabetic subjects ${ }^{(7,11,14,24-26)}$. Up to now, only one study investigated the effect of a diet enriched in $\beta$-glucan on HbA1c in type 2 diabetes, in addition to the recommended American Diabetes Association diet ${ }^{(14)}$. Eight diabetic patients (mean HbA1c of $8.5 \%$ ) followed a 4-week low-energy diet, enriched with $8 \%$ of $\beta$-glucan. Fasting glycaemia did not change, but a weak decrease in HbA1c was observed in the $\beta$-glucan group. But $\beta$-glucan enrichment was associated to the replacement of sugars by sweeteners. In our diabetic group, the lower baseline HbAlc (7.38 (SD 1.10) \%) may explain the lack of significant effect of soups used as the exclusive intervention. Another fibre, konjac-mannan, significantly decreased fructosamine in diabetic subjects ${ }^{(23)}$. In the present study, fructosamine analysis was performed between W0 and W11 (data not shown), and no change was observed.

Concerning fasting glycaemia, we did not found any significant effect of $\beta$-glucan. There was a trend for a decrease in fasting glycaemia in subgroup without insulin injection (NS). Aro et al. ${ }^{(21)}$ showed a significant decrease in fasting and post-prandial hyperglycaemia in type 2 diabetic subjects following a 3-month supplementation (three times $7 \mathrm{~g}$ guar gum daily).

Considering study design using dietary supplementation in free-living subjects, discrepancies between results can thus partly be explained by differences in food matrix, amount of $\beta$-glucan ingested, ingestion frequency, HbA1c level and lipid status at baseline, intervention duration and population size. It is well known that the fibre type, the interactions with other constituents in the composite food matrix or the mode of administration are likely to influence structural features, concentration and solubility of $\beta$-glucans and consequently modulate their physiological action. The efficiency of $\beta$-glucan to alter metabolic parameters such as cholesterol level is higher when $\beta$-glucan is part of a liquid food matrix compared to a solid one ${ }^{(10,16,18,27)}$. Moreover, one study has been done in order to verify the willingness of consumers to use beverages and ready-to-eat frozen soups containing oat $\beta$-glucan ${ }^{(28)}$. Although $\beta$-glucan addition altered the sensory characteristics of both the beverages and soups, willingness to use soups was not remarkably lowered. Whereas soups seemed to be a good vector of administration, well tolerated, palatable and convenient, the acceptable amount of $\beta$-glucan $(3.5 \mathrm{~g} / \mathrm{serving})$ may not be sufficient to induce a physiological effect on lipid or glucose profile. More than $3.5 \mathrm{~g}$ per serving, we encountered high decrease in palatability, which could have compromised the good compliance to the study and a possible future extension in the longer-term. Finally, the frequency of ingestion may have negatively influenced the present results since the $\beta$-glucan soup was ingested only once a day compared to other studies ${ }^{(16,23)}$. Besides, $\beta$-glucan soups were ingested as main part of a meal and may have replaced other fibre-rich food as vegetables or fruits in the dietary pattern.

In conclusion, this 2-month intervention trial in a large group of type 2 diabetic subjects shows that a single daily ingestion of a soup enriched with $3.5 \mathrm{~g} \beta$-glucan (according to official recommendations) does not improve the lipid profile and HbA1c level in free-living conditions. Long-term benefits from $\beta$-glucan-enriched foods in the diabetic population might occur with higher doses and/or increased frequency of intake, but such consideration raises the question of the supplementation acceptability.

\section{Acknowledgements}

The present work was a part of the European project QLKI2000-00535 'Design of food with improved functionality and superior health effects using cereal $\beta$-glucans' supported by the European Commission. It does not necessarily reflect its views and in no way anticipates the Commission's future policy in this area. Additional support was obtained from the Påhlsson Foundation and the Swedish Governmental Agency for Innovation Systems (to G. O.). J. H. is employee of Findus.

We thank all the staff of the Centre de Recherche en Nutrition Humaine Rhône-Alpes and of the Unit for diabetic studies (Lund University) for their excellent technical assistance in subject recruitment, sample collection and analysis, and dietary surveys, Dr T. Suortti, Dr M. Salmenkallio-Marttila from VTT Biotechnology for analysis of the study products, Findus R\&D for the frozen soups production, Picard Surgelés (France) for the frozen soups supply and all the subjects for taking part in this trial.

C. C.-A. and J.-A. N. were responsible for data collection and analysis, statistical analysis, writing and evaluation of the paper. M. B. and E. L. C. were responsible for study coordination, data collection and analysis. A. S. was responsible for biochemical assays. M. S. was responsible for dietary surveys and analysis. J. H. worked at Findus and provided expertise and knowledge in test products. M. L.-O. was responsible for subject involvement and coordination of subject management. M. L., G. O. and P. M. were responsible for study coordination, study design and supervised the writing of the paper.

\section{References}

1. Salmeron J, Ascherio A, Rimm EB, et al. (1997) Dietary fiber, glycemic load, and risk of NIDDM in men. Diabetes Care 20, $545-550$

2. Wolk A, Manson JE, Stampfer MJ, et al. (1999) Long-term intake of dietary fiber and decreased risk of coronary heart disease among women. JAMA 281, 1998-2004.

3. Liu S, Buring JE, Sesso HD, et al. (2002) A prospective study of dietary fiber intake and risk of cardiovascular disease among women. J Am Coll Cardiol 39, 49-56.

4. Braaten JT, Wood PJ, Scott FW, et al. (1991) Oat gum lowers glucose and insulin after an oral glucose load. Am J Clin Nutr 53, $1425-1430$.

5. Wursch P \& Pi-Sunyer FX (1997) The role of viscous soluble fiber in the metabolic control of diabetes. A review with special emphasis on cereals rich in beta-glucan. Diabetes Care $\mathbf{2 0}$, 1774-1780.

6. Brown L, Rosner B, Willett WW, et al. (1999) Cholesterollowering effects of dietary fiber: a meta-analysis. Am J Clin Nutr 69, 30-42.

7. Tappy L, Gugolz E \& Wursch P (1996) Effects of breakfast cereals containing various amounts of beta-glucan fibers on 
plasma glucose and insulin responses in NIDDM subjects. Diabetes Care 19, 831-834.

8. Lia A, Hallmans G, Sandberg AS, et al. (1995) Oat beta-glucan increases bile acid excretion and a fiber-rich barley fraction increases cholesterol excretion in ileostomy subjects. Am J Clin Nutr 62, 1245-1251.

9. Keogh GF, Cooper GJ, Mulvey TB, et al. (2003) Randomized controlled crossover study of the effect of a highly beta-glucanenriched barley on cardiovascular disease risk factors in mildly hypercholesterolemic men. Am J Clin Nutr 78, 711-718.

10. Naumann E, van Rees AB, Onning G, et al. (2006) Beta-glucan incorporated into a fruit drink effectively lowers serum LDLcholesterol concentrations. Am J Clin Nutr 83, 601-605.

11. Battilana P, Ornstein K, Minehira K, et al. (2001) Mechanisms of action of beta-glucan in postprandial glucose metabolism in healthy men. Eur J Clin Nutr 55, 327-333.

12. Wood PJ, Braaten JT, Scott FW, et al. (1994) Effect of dose and modification of viscous properties of oat gum on plasma glucose and insulin following an oral glucose load. Br J Nutr 72, $731-743$.

13. Jenkins DJ, Kendall CW, Vuksan V, et al. (2002) Soluble fiber intake at a dose approved by the US Food and Drug Administration for a claim of health benefits: serum lipid risk factors for cardiovascular disease assessed in a randomized controlled crossover trial. Am J Clin Nutr 75, 834-839.

14. Reyna NY, Cano C, Bermudez VJ, et al. (2003) Sweeteners and beta-glucans improve metabolic and anthropometrics variables in well controlled type 2 diabetic patients. Am J Ther 10, 438-443.

15. Friedewald WT, Levy RI \& Fredrickson DS (1972) Estimation of the concentration of low-density lipoprotein cholesterol in plasma, without use of the preparative ultracentrifuge. Clin Chem 18, 499-502.

16. Onning G, Wallmark A, Persson M, et al. (1999) Consumption of oat milk for 5 weeks lowers serum cholesterol and LDL cholesterol in free-living men with moderate hypercholesterolemia. Ann Nutr Metab 43, 301-309.

17. Torronen R, Kansanen L, Uusitupa M, et al. (1992) Effects of an oat bran concentrate on serum lipids in free-living men with mild to moderate hypercholesterolaemia. Eur J Clin Nutr 46, 621-627.
18. Kerckhoffs DA, Hornstra G \& Mensink RP (2003) Cholesterollowering effect of beta-glucan from oat bran in mildly hypercholesterolemic subjects may decrease when beta-glucan is incorporated into bread and cookies. Am J Clin Nutr 78, 221-227.

19. Biorklund M, Holm J \& Onning G (2008) Serum lipids and postprandial glucose and insulin levels in hyperlipidemic subjects after consumption of an oat beta-glucan-containing ready meal. Ann Nutr Metab 52, 83-90.

20. Ripsin CM, Keenan JM, Jacobs DR Jr, et al. (1992) Oat products and lipid lowering. A meta-analysis. JAMA 267, 3317-3325.

21. Aro A, Uusitupa M, Voutilainen E, et al. (1981) Improved diabetic control and hypocholesterolaemic effect induced by long-term dietary supplementation with guar gum in type 2 (insulin-independent) diabetes. Diabetologia 21, 29-33.

22. Pick ME, Hawrysh ZJ, Gee MI, et al. (1996) Oat bran concentrate bread products improve long-term control of diabetes: a pilot study. J Am Diet Assoc 96, 1254-1261.

23. Vuksan V, Jenkins DJ, Spadafora P, et al. (1999) Konjacmannan (glucomannan) improves glycemia and other associated risk factors for coronary heart disease in type 2 diabetes. A randomized controlled metabolic trial. Diabetes Care 22, 913-919.

24. Braaten JT, Scott FW, Wood PJ, et al. (1994) High beta-glucan oat bran and oat gum reduce postprandial blood glucose and insulin in subjects with and without type 2 diabetes. Diabet Med 11, 312-318.

25. Brennan CS (2005) Dietary fibre, glycaemic response, and diabetes. Mol Nutr Food Res 49, 560-570.

26. Nazare JA, Normand S, Oste Triantafyllou A, et al. (2008) Modulation of the postprandial phase by beta-glucan in overweight subjects: effects on glucose and insulin kinetics. Mol Nutr Food Res 53, 361-369.

27. Anderson JW, Davidson MH, Blonde L, et al. (2000) Long-term cholesterol-lowering effects of psyllium as an adjunct to diet therapy in the treatment of hypercholesterolemia. Am J Clin Nutr 71, 1433-1438.

28. Lyly M, Honkapää K, Poutanen K, et al. (2007) Factors influencing consumers' willingness to use beverages and ready-to-eat frozen soups containing oat beta-glucan in Finland. Food Qual Prefer 18, 242-255. 